\title{
2013 Proceedings
}
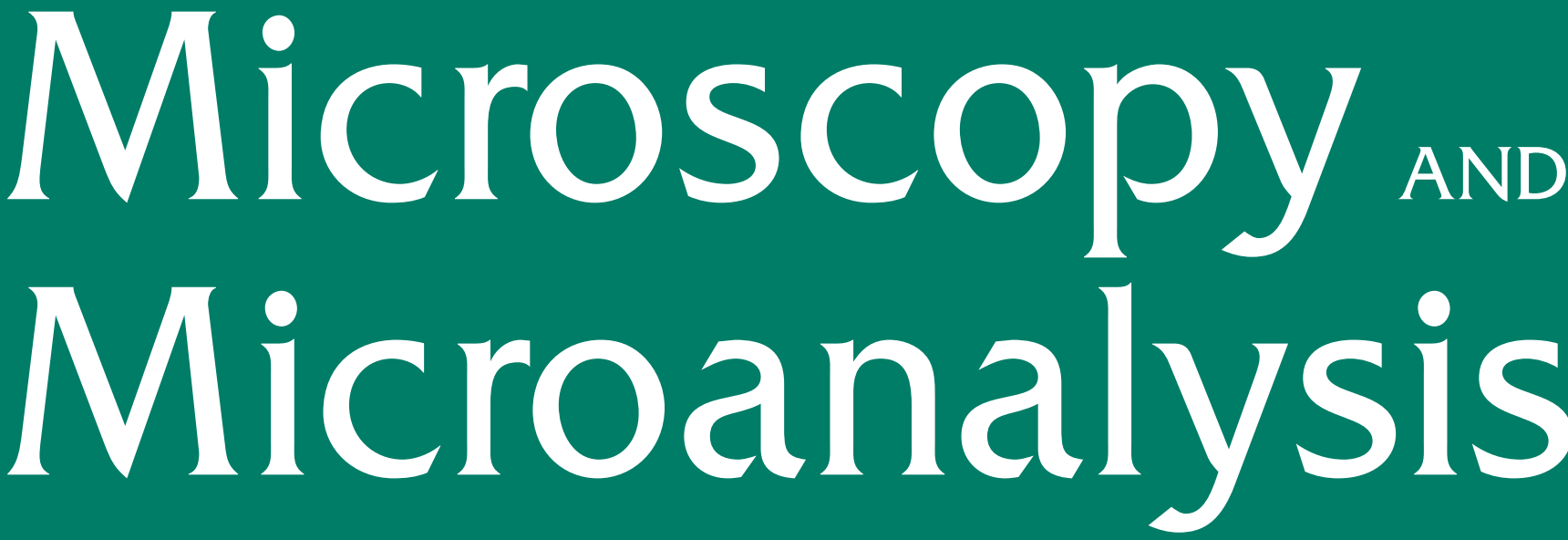

\section{Part 3: Advances in Instrumentation}

and Techniques Symposia-II

Indianapolis, Indiana, USA

August 4-8, 2013

Microscopy Society of America

71st Annual Meeting

N. Zaluzec

T. Ruiz

D. Bell

E. Marquis

C. McNee
International Metallographic Society 46th Annual Meeting

Published By

Cambridge University Press 


\title{
Method Development for Chemical Profiling of a Cell Population Using MALDI Mass Spectrometry Imaging
}

\author{
Ta-Hsuan Ong, David Kissick, Stanislav Rubakhin, and Jonathan Sweedler \\ University of Illinois at Urbana-Champaign, Department of Chemistry, Urbana, USA
}

The chemical heterogeneity between individual cells is a fundamental aspect of organism function [1]. Not only do variations exist among different kinds of cells, a supposed homogenous population also often contains surprising variability in cellular chemical profile. While studies on bulk number of cells can generate an average of the chemical content in a cell population, single-cell studies are important for characterizing these systems in detail. Flow cytometry is a well-established technique for single-cell studies, but because detection typically relies on molecular recognition approaches, analyte pre-selection limits the obtainable chemical information. In this study, we create a workflow combining optical microscopy and matrix assisted laser desorption ionization mass spectrometry imaging (MALDI-MSI) to conduct information-rich single cell studies on cell populations (Figure 1).

A preliminary study using MSI to profile individual cells in a population has been conducted on rat dorsal root ganglion cells (Figure 2), which shows that MALDI-MSI possesses the necessary detection limit to profile single cell chemical content and find rare cells. In this work, rather than imaging the entire slide by rastering the MALDI laser as is typical for an MSI experiment, we seek to limit data acquisition to only cell soma locations. As illustrated in Figure 1, we start by imaging the slide with optical microscopy to determine the locations of the cell soma. These locations are then loaded into our mass spectrometer to automatically acquire mass spectra only from intact soma. By doing so, both analysis time and data file size are reduced.

To demonstrate our ability to probe only cell locations, the workflow was tested on $50 \mu \mathrm{m} 2,5$, dihydroxybenzoic acid (DHB) spots printed at random locations on a slide (ChIP, Shimadzu, Tokyo, Japan). A brightfield mosaic image of the slide was taken (AxioVert 200, Carl Zeiss, Jena, Germany) and stitched together using an ImageJ plugin [2]. The spots' centroid coordinates in terms of image pixels were then obtained using the "Analyze Particles" plugin in ImageJ. Once the coordinates were found, a custom geometry file for the MALDI-TOF mass spectrometer (UltrafleXtreme, Bruker Daltonics, Germany) was generated using a previously developed in-house program [3]. Using specifically set reference points, this program converts the optical image coordinates to the coordinate system used by the mass spectrometer. Spatial accuracy of the geometry file was visually determined using the mass spectrometer's sample camera, and a spectrum could be obtained from each spot using the smallest instrument laser beam footprint size $(\sim 50 \mu \mathrm{m}$ in diameter; measured by burning through a thin layer of alpha-cyano-4-hydroxycinnamic acid). Subsequence mass spectrometry image generation was done using a Java code developed previously.

To apply the workflow to cells, cell populations consisting of bag cell neurons and pleural sensory neurons from Aplysia californica were dispersed onto a slide. Brightfield microscopy was found to have insufficient contrast for cells for image analysis. Therefore, darkfield and autofluorescence images were taken. To ensure consistent registry between the two images and to reduce imaging time, both images were taken simultaneously on separate channels of a color CCD camera. The darkfield image was restricted to the red channel via a red filter. Autofluorescence imaging was conducted with $365 \mathrm{~nm}$ 
excitation using a mercury vapor lamp. Emission occurred mostly below $500 \mathrm{~nm}$ and was taken on the blue channel of the camera. Cell coordinates were then calculated by examining the overlap between the blue and red channels.

The obtained coordinates were then converted to a custom geometry file in the same fashion as for the DHB spots. Matrix was applied using a sublimation and recrystallization method to create matrix crystals that are around $1 \mu \mathrm{m}$ in size [4]. The small crystal size is important for imaging small features such as cells because it minimizes analyte delocalization. Principal component analysis allows us to discriminate between the two distinct cell types. Future work will examine the differences between and within more homogenous cell populations and examine the cells that have unusual chemical profiles.

\section{References:}

[1] S. Rubakhin et al, Nature Methods 8 (2011), p. S20-S29.

[2] S Preibisch et al, Bioinformatics 25 (2009), p. 1463.

[3] T Zimmerman et al, Analytical Chemistry 81 (2009), p. 9402.

[4] J Yang, R Caprioli, Analytical Chemistry 83 (2011), p. 5728.

[5] The authors acknowledge funding from the National Institutes of Health through 1R21MH10070401 and the National Science Foundation through CHE-11-11705.

A

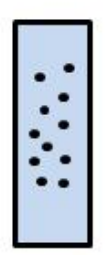

B

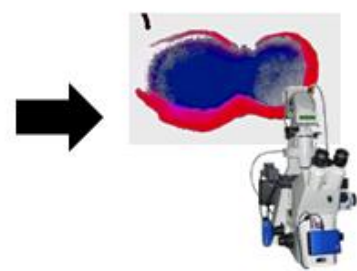

C

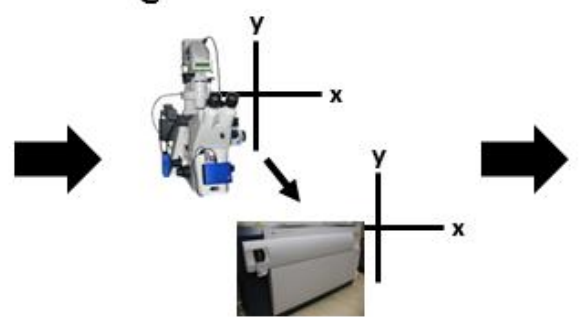

D

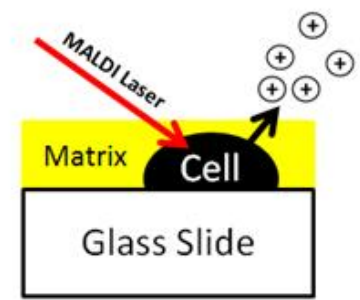

Figure 1. Workflow for chemical profiling of single cells in populations using MALDI-MSI. A. Cell populations are dispersed onto a slide. B. Simultaneous autofluorescence (blue) and darkfield (red) imaging (inset image modified to enhance contrast). C. Geometry file generation using ImageJ and an in-house written program. D. Mass spectrometry analyses are performed only at cell locations.
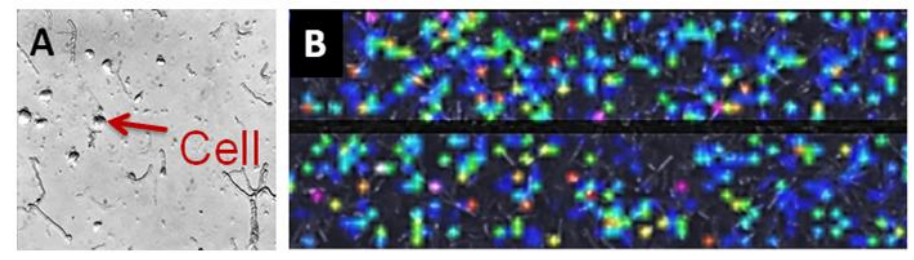

Figure 2. MSI profiling of dispersed rat dorsal root ganglion cells on ITO slide. A) Microphotograph of sample region. B) Image depicting distribution of m/z 1280. 